\title{
Déficit hétérozygote et homozygote en p53 provoqué par recombinaison homologue chez la souris
}

La protéine p53 est maintenant familière aux lecteurs de $\mathrm{m} / \mathrm{s}$. A l'état normal, elle semble douée de propriétés anti-prolifératives mais, en cas de mutation, peut se révéler avoir des effets oncogéniques. Au cours du cycle cellulaire, la localisation et l'état de phosphorylation de $\mathrm{p} 53$ se modifient : p53 est, en effet, un substrat de la protéine kinase liée au cycle, $\mathrm{p} 34^{\mathrm{cdc} 2}$, et est donc phosphorylée de façon maximale durant la mitose. Par ailleurs, p53 s'accumule dans le cytoplasme en phase $\mathrm{G} 1$ et migre dans le noyau en phase $\mathrm{S}$. Le mode d'action précis de p53 n'est pas exactement connu. Beaucoup d'arguments indiquent qu'elle pourrait être un facteur transcriptionnel contrôlant négativement l'expression d'oncogènes $(\mathrm{m} / \mathrm{s}$ $n^{\circ} 2$, vol. 8, p. 184) ou positivement l'expression d'anti-oncogènes. Par ailleurs, on sait que p53 peut se fixer à différents oncogènes viraux, l'antigène $\mathrm{T}$ de $\mathrm{SV} 40$ et l'oncogène $E 1 B$ d'adénovirus ( $\mathrm{m} / \mathrm{s} n^{\circ} 8$, vol. 5 , p. 598 et $n^{\circ} 8$, vol. $\left.6, p .821\right)$. Le gène $p 53$ est altéré dans près de $55 \%$ des tumeurs humaines, ce qui constitue par conséquent l'anomalie moléculaire la plus fréquente $\left(\mathrm{m} / \mathrm{s} n^{\circ} 3\right.$, vol. $8, \quad$ p. 289). Il existe une affection congénitale, à transmission dominante, de prédisposition au cancer secondaire à une mutation constitutionnelle d'un allèle du gène $p 53$, le plus souvent dans l'exon $\mathrm{n}^{\circ} 7$ : le syndrome de Li Fraumeni ( $\mathrm{m} / \mathrm{s} n^{\circ} 10$, vol. 6, p. 1006). C'est pour préciser le rôle de p53 dans le développement et pour créer des modèles animaux de prédisposition au cancer que l'équipe d'Allan Bradley (Baylor College, Houston, TX, USA) [1] a créé des souris transgéniques dont un ou deux allèles du gène p53 ont été inactivés par recombinaison homologue. A l'état hétérozygote, les animaux se développent parfaite- ment bien et n'ont qu'exceptionnellement des cancers (de l'ordre de $2 \%$ ). En cela, ces animaux ne sont pas de vrais modèles du syndrome de $\mathrm{Li}$ Fraumeni. Dans cette maladie humaine, cependant, la lésion moléculaire est presque toujours une mutation ponctuelle pouvant entraîner l'accumulation d'une protéine p53 oncogénique, ce qui pourrait expliquer la plus grande incidence de cancers que chez un animal ayant un allèle muet. Une telle situation d'un allèle muet $a$, néanmoins, été notée chez un malade atteint du syndrome de Li Fraumeni. Une autre explication des différences de susceptibilité au cancer chez les souris hétérozygotes et chez les malades atteints du syndrome de Li Fraumeni est, évidemment, la longévité pouvant, chez l'homme, augmenter considérablement les risques de survenue d'une altération complémentaire activant l'allèle initialement non modifié de p53.

Chez les homozygotes, en revanche, un cancer est observé chez plus de $70 \%$ des animaux, survenant le plus souvent précocement $(74 \%$ de tumeurs à 6 mois). Les lymphomes constituent les proliférations les plus souvent observées chez ces souris p $53^{-1-}$, ce qui contraste avec la diversité des cancers décrits chez les malades atteints du syndrome de $\mathrm{Li}$ Fraumeni. Il pourrait se faire que la lignée de souris utilisée pour ces expériences de recombinaison homologue, naturellement sensible au développement tardif de lymphomes, fût responsable de ces résultats. Dans ce cas, la mutation de p53 accélérerait et augmenterait la fréquence d'un phénomène auquel les souris seraient, génétiquement, prédisposées. Il faut noter que les tumeurs qui se développent chez les animaux homozygotes restent clonales, indiquant que l'absence de p53 ne dispense pas de la nécessité d'altérations génétiques supplémentaires, aléatoires, pour que se développe une prolifération maligne invasive. En réalité, le résultat le plus surprenant de cette étude est que le développement embryonnaire de ces animaux dépourvus de toute synthèse de p53 soit normal. Cela signifie que, malgré ses modifications au cours du cyle cellulaire, p53 n'est pas indispensable aux mitoses et au développement. Cette protéine pourrait, plutôt, jouer un rôle de contrôle négatif redondant de la prolifération cellulaire, constituant, en quelque sorte, une double sécurité contre la prolifération incontrôlée. En ce sens, p53 joue un rôle tout différent du produit du gène de susceptibilité au rétinoblastome, p105 Des animaux homozygotes pour une inactivation du gène $R b$ par recombinaison homologue ont, en effet, été obtenus récemment... d'après de nombreuses informations de "couloirs de congrès ". p105 ${ }^{\mathrm{Rb}}$, comme p53, est étroitement liée au cycle cellulaire, sa phosphorylation par p34 $4^{\text {cdc2 }}$ semblant tout à fait indispensable à la mitose. De fait, les souris homozygotes $\mathrm{Rb}^{-1-}$ présenteraient des troubles majeurs du développement embryonnaire, ne permettant pas la naissance d'animaux viables. Nous ne savons pas encore si les animaux hétérozygotes pour l'inactivation d'un allèle du gène $R b$ présentent, quant à eux, une prédisposition au développement de tumeurs, telles qu'on le voit dans les cas de rétinoblastomes familiaux.

A. $\mathbf{K}$.

1. Donehower LA, Harvey M, Slagle PL, et al. Mice deficient for p53 are developmentally normal but susceptible to spontaneous tumors. Nature $1992 ; 356: 215-21$. 